\title{
L'engagement des entreprises dans le dispositif de formation professionnelle en Allemagne
}

The engagement of business in Germany's vocational training system

El compromiso de las empresas en el dispositivo de formación profesional en

Alemania

Isabelle Le Mouillour et Marthe Geiben

\section{OpenEdition}

\section{Journals}

Édition électronique

URL : https://journals.openedition.org/ries/4606

DOI : $10.4000 /$ ries.4606

ISSN : 2261-4265

Éditeur

France Education international

Édition imprimée

Date de publication : 1 avril 2016

Pagination : 63-72

ISBN : 978-2-85420-610-4

ISSN : 1254-4590

Référence électronique

Isabelle Le Mouillour et Marthe Geiben, «L'engagement des entreprises dans le dispositif de formation professionnelle en Allemagne », Revue internationale d'éducation de Sèvres [En ligne], 71 | avril 2016, mis en ligne le 01 avril 2018, consulté le 02 juillet 2021. URL : http://journals.openedition.org/ries/4606 ; DOI : https://doi.org/10.4000/ries.4606 


\title{
L'engagement des entreprises dans le dispositif de formation professionnelle en Allemagne
}

\author{
Isabelle Le Mouillour \\ Institut Fédéral Allemand \\ de la formation professionnelle (BIBB) \\ Marthe Geiben \\ Institut Fédéral Allemand \\ de la formation professionnelle (BIBB)
}

Depuis quelques années, le modèle de formation professionnelle duale connaît un regain d'intérêt qui semble être justifié par le faible taux de chômage que connaissent les diplômés du système dual en Allemagne. Les liens institutionnalisés entre les acteurs publics du système de formation et les acteurs du monde professionnel semblent jouer en faveur d'une meilleure orientation de la formation vers les besoins du marché du travail. L'Allemagne compte environ 438000 entreprises actives en formation professionnelle initiale, dont une large proportion de PME (environ $97 \%$ des entreprises formatrices ont moins de 249 salariés ; 49 \% moins de 9 salariés), 20,7 \% des entreprises allemandes participaient ainsi à la formation duale de niveau initial en 2013 (BIBB, 2015).

Le système de formation professionnelle duale en Allemagne comptait environ 1,5 million d'apprentis dans 327 métiers à formations réglementées (Ausbildungsberufe) qui mènent à des postes d'employés et d'ouvriers qualifiés en 2014 (BIBB, 2015). Les acteurs du monde professionnel comprennent, dans le système allemand, les entreprises et les organisations les représentant (par exemple, la Fédération nationale des organisations patronales allemandes) ou les chambres consulaires ainsi que les organisations syndicales, comme la Fédération des syndicats allemands.

L'État et les acteurs de monde du travail partagent la responsabilité de la définition des règlements de formation, de la mise en place, de l'organisation et du contrôle de la formation duale ainsi que de son financement. Une autre responsabilité partagée se trouve au niveau juridique, en respect des principes du fédéralisme en matière d'éducation et de la Loi fondamentale (Grundgesetz) : l'État fédéral (Bund) et les Länder ont différentes responsabilités dans le système de formation professionnelle, et celles-ci sont fixées dans le cadre de la loi fédérale sur la formation professionnelle. Tandis que l'État fédéral est responsable des éléments cadres de la formation en entreprise, les Länder sont responsables 
de la formation en milieu scolaire. On parle d'une dualité visible dans plusieurs domaines : deux lieux d'apprentissage (l'entreprise pour environ 60 à $70 \%$ et l'école professionnelle pour 30 à $40 \%$ du temps de formation), un financement assuré par l'État et les entreprises, des bases juridiques doubles en raison du principe de subsidiarité en matière de formation scolaire, avec la loi de 2005 sur la formation professionnelle au niveau national (Berufsbildungsgesetz) et les lois scolaires des Länder, un modèle de gouvernance partagée entre l'État et les organisations représentant le monde professionnel (employeurs, syndicats).

Nous abordons dans cet article les différents aspects de l'intervention et de la participation des acteurs du monde professionnel dans le système de formation duale en Allemagne. Nous considérons ici le système de formation professionnelle duale initiale, qui accueille en Allemagne une majorité des jeunes au niveau du deuxième cycle du secondaire (à partir d'environ 16 ans) ${ }^{1}$ et qui mène à des certifications de niveau 2 à 4 du cadre allemand de certifications ${ }^{2}$. Plus de $50 \%$ d'une classe d'âge a choisi en 2013 une formation professionnelle de type dual, dont environ $25 \%$ détenteurs d'un baccalauréat ou d'une certification équivalente (BIBB, 2015).

\section{LE SYSTÈME DE FORMATION PROFESSIONNELLE DUALE}

La formation initiale duale (apprentissage) en Allemagne est réglée par la loi sur la formation professionnelle de 2005 (Berufsbildungsgesetz) et le code de l'artisanat (Handwerksordnung) ; le code a été révisé pour la dernière fois en 2004 pour prendre en compte les développements européens. Martinot (2015) souligne dans son analyse comparative la stabilité des textes législatifs en matière de formation professionnelle, puisque la loi fédérale fondatrice date de 1969 et n'a été révisée qu'une seule fois en 2005. Elle prévoit une durée générale de formation entre 2 et 3 ans. L'apprenti et l'entreprise formatrice signent pour la durée de la formation un contrat de formation, et ce contrat fait de l'apprenti un membre du personnel de l'entreprise, avec tous les droits et devoirs qui lui sont liés (rémunération, cotisation d'assurance maladie et vieillesse, vacances, etc.). L'entreprise s'oblige à respecter les heures de scolarité, le plan de formation en entreprise et doit veiller à ce que l'apprenti aille en école professionnelle, au titre de la loi sur la protection des mineurs et de la loi sur le temps de travail.

Pour comprendre le fonctionnement du système de formation duale et la place du monde professionnel dans ce système, il est nécessaire de se pencher sur certaines caractéristiques et principes fondamentaux qui guident l'action de tous les acteurs de la formation professionnelle, garantissent sa qualité et sont codifiés dans la loi sur la formation professionnelle:

\footnotetext{
1. Des programmes au premier cycle du secondaire sont des programmes de formation générale uniquement. Ici, il y a des programmes d'orientation comme des stages en entreprises par exemple ou la « découverte d'une profession », qui sont intégrés dans les matières générales.

2. Les niveaux du cadre allemand de certifications sont équivalents à ceux du cadre européen de certification.
} 
Le principe de consensus. Ce dernier exprime la volonté et la capacité d'établir un compromis entre les associations patronales, les syndicats et la Fédération sur les questions de formation professionnelle. La coopération entre les différents acteurs est institutionnalisée dans le comité principal de l'Institut fédéral de la formation professionnelle (BIBB), parfois également qualifié de «parlement» de la formation professionnelle, et dans le processus de développement et d'actualisation des règlements de formation (voir infra). Le principe de consensus a sous-tendu l'action commune qui a mené en 2004 le gouvernement fédéral et les principales fédérations représentatives des entreprises à conclure pour la première fois le «pacte national pour la formation et la relève en main d'œuvre qualifiée », qui prévoyait une intensification de l'engagement de tous les acteurs de la formation professionnelle. Reconduit en 2010, ce pacte est devenu en 2014 une " alliance pour la formation initiale et continue » pour améliorer la situation sur le marché de la formation pour les jeunes et les entreprises. Cette alliance est portée par l'État fédéral, les organisations représentatives des syndicats et des entreprises et les Länder.

Le principe de responsabilité commune à plusieurs niveaux. Il fait référence au partenariat entre l'entreprise et l'école professionnelle dans le système dual face à leur obligation d'agir pour la réussite de l'apprenti. En outre, il comprend la responsabilité commune de l'État et des organisations du monde professionnel ainsi que la responsabilité commune de la Fédération et des Länder.

Le principe "Beruf » ou la référence à un métier. La loi sur la formation professionnelle allemande définit l'apprentissage par son but : «transmettre les compétences, connaissances et capacités professionnelles nécessaires à l'exercice d'une activité professionnelle qualifiée dans un monde du travail en mutation » (Loi sur la formation professionnelle, 2005). Cette approche par le métier inclut le développement de la personnalité et l'insertion sociale et pose les bases pour un apprentissage tout au long de la vie. Ce principe se retrouve dans les règlements de formation dans une profession sanctionnée par un diplôme d’État (métier). Le métier est également facteur d'identité collective, en ce qu'il assure une forte lisibilité des compétences acquises sur un marché du travail par ailleurs très segmenté (Lasserre, 2014).

Validité nationale des règlements. Les règlements de formation pour une profession sanctionnée par un diplôme d'État sont des règlements cadres ayant une validité dans toute l'Allemagne, tout en laissant une certaine marge pour une adaptation aux contextes économiques et industriels régionaux. Le contenu de ces règlements doit être transmis pendant la formation et est examiné à la fin de la formation initiale. Les examens mènent à des diplômes reconnus nationalement, délivrés par les chambres consulaires.

Formation professionnelle en milieu de travail. La formation professionnelle se déroule dans une vaste majorité en entreprises ou au sein de réseaux d'entreprises. Les règlements de formation pour une profession sanctionnée par un diplôme d'État élaborés pour les 327 métiers fixent le contenu de la formation en milieu de travail. 
Personnel formateur qualifié. Les entreprises sont soumises à certaines réglementations pour être habilitées à offrir une place d'apprentissage. L'une d'elles est l'obligation d'employer un collaborateur titulaire de la qualification reconnue de formateur par apprentissage (technicien/ contremaître/ maître artisan). Les contenus de cette formation sont fixés dans le règlement sur l'aptitude des tuteurs.

Recherche institutionnalisée, orientée vers les besoins exprimés par la mise en œuvre de la politique et des pratiques en formation professionnelle. La recherche institutionnalisée est réalisée en grande partie par l'Institut fédéral de la formation professionnelle (BIBB). Ses résultats sont publiés sous forme de rapport annuel sur la formation professionnelle, de guides pédagogiques pour la mise en pratique des référentiels; les projets de recherche font l'objet de discussion avec les acteurs du monde professionnel et sont organisés dans le cadre du programme annuel de recherche du BIBB. Le BIBB travaille de concert avec l'Institut fédéral d'analyse du marché du travail (IAB), un centre de recherche rattaché à l'Agence fédérale pour l'emploi.

\section{RESPONSABILITÉ PARTAgÉE}

En Allemagne, le système de l'apprentissage repose sur un texte législatif majeur élaboré en 1969 et révisé en 2005. La loi se contente d'énoncer les principes généraux sans lesquels il n'y aurait pas de système national cohérent d'apprentissage : règlements de formation (similaires aux référentiels de certification en France), contenu des examens, droit du contrat de travail (durée, période d'essai, temps de travail, conditions de travail des mineurs), coordination et répartition des compétences entre les acteurs. Elle régit également le fonctionnement et les missions de la tête de réseau du système (BIBB), ainsi que les principes fondamentaux des remontées statistiques s'imposant à tous les acteurs. La loi laisse une certaine latitude aux Länder et aux entreprises formatrices pour l'adaptation de la réglementation aux réalités professionnelles et régionales : organisation et financement des écoles professionnelles, règles régissant les conditions d'accès au titre de maître d'apprentissage, contrôle de l'apprentissage (Martinot, 2015).

La Loi fédérale sur la formation professionnelle en Allemagne règle la responsabilité des différents acteurs et les mécanismes de codécision. La responsabilité partagée tripartite passe par un partage des tâches selon le schéma suivant :

- les partenaires sociaux (les syndicats de salariés et associations patronales) négocient le montant de l'indemnité d'apprentissage, contribuent à définir des règlements de formation professionnelle et siègent dans les commissions des examens organisées par les chambres consulaires ; les conseils d'entreprise supervisent la formation en entreprise ;

- l'État finance, supervise et contrôle le système de formation professionnelle publique, organise la recherche institutionnalisée sur la formation, veille à actualiser les règlements de formation et assiste les jeunes sans emploi, 
défavorisés ou affectés d'un handicap dans leur recherche de formation ; en outre, il apporte une aide à l'orientation professionnelle et fait mieux connaître la formation professionnelle duale ;

- les chambres consulaires ${ }^{3}$ agissent comme des organisations intermédiaires : elles conseillent les entreprises formatrices, forment le personnel formateur, accréditent ou habilitent les entreprises formatrices, contrôlent la formation en entreprises (équipements, formateurs, etc.), assistent les entreprises dans leur recherche d'apprentis, enregistrent les contrats de formation, organisent les examens partiels et finaux et sont médiateurs en cas de conflit entre l'apprenti et l'entreprise. Ce sont les chambres qui délivrent les diplômes du système de formation duale. De fait, la responsabilité pour la qualité de la formation professionnelle en entreprise est déléguée aux chambres consulaires.

La responsabilité partagée et la codécision en Allemagne sont institutionnalisées dans le comité principal (Hauptausschuss) du BIBB. Sa composition est fixée par la loi sur la formation professionnelle. S’y retrouvent des délégués des employeurs, des salariés et des Länder en nombre identique ainsi que des délégués des ministères fédéraux compétents. Un délégué ou une déléguée de l'Agence fédérale pour l'emploi, des associations de communes au niveau fédéral ainsi que du conseil scientifique du BIBB peuvent participer aux réunions du comité principal avec une voix consultative. Les missions du comité principal sont entre autres le conseil auprès du gouvernement fédéral sur les questions fondamentales relatives à la formation professionnelle. De plus, il décide du programme annuel de recherche du BIBB, peut émettre des recommandations sur l'application uniforme de la loi sur la formation professionnelle et un avis sur les projets de règlements de formation préparés au BIBB.

\section{FINANCEMENT DE LA FORMATION PROFESSIONNELLE}

Le système de formation professionnelle duale repose entre autres sur un partage des responsabilités entre le gouvernement fédéral et les gouvernements des 16 Länder. Le financement de la partie de la formation organisée dans les écoles professionnelles est de façon générale assuré par les Länder ; ces derniers prennent en charge les frais liés aux bâtiments, équipements et personnel enseignant. Les entreprises prennent en charge les frais liés à la formation en entreprises, ce qui comprend les frais d'équipement des apprentis (vêtements, protection, outils, etc.), les matériaux et machines, ainsi que la rémunération des apprentis (charges sociales incluses) et de leurs formateurs. Les entreprises ne sont cependant pas tenues de former ni de participer, comme en France, à un dispositif de mutualisation financière de l'effort de formation (Lasserre, 2014). Les financements publics

3. En Allemagne, les entreprises ont obligation d'être membres d'une chambre. 
en Allemagne tendent à se concentrer sur les jeunes en difficulté ${ }^{4}$. Ils prennent également la forme de programmes et initiatives spécifiques pour les petites et moyennes entreprises, par exemple pour la formation de centres interentreprises, et sont mobilisés de manière importante dans le cadre du soutien au développement économique et social. L'État assure le financement des frais de modernisation des référentiels de formation depuis les travaux de recherche jusqu'à leur publication, ainsi que la grande majorité des travaux de recherche effectués au BIBB. Les dépenses publiques pour la formation professionnelle en milieu scolaire s'élèvent à environ 7,6 milliards d'euros au total en 2014 ; 6,9 milliards d'euros sont utilisés pour le financement des 8855 écoles professionnelles, dont 1559 écoles participant au système de formation duale pour un budget de 2,9 milliards d'euros. Les 0,6 milliard d'euros restants financent des mesures fiscales, de contrôle et d'aide (BIBB, 2015 ; Statistisches Bundesamt, 2015).

La participation financière des entreprises au système de formation duale est largement considérée comme des frais d'investissement. En moyenne, les coûts bruts pour une entreprise s'élèvent à environ $17933 €$ par an et par apprenti. La rémunération des apprentis est le poste le plus important dans ce calcul, puisqu'il représente en moyenne $61 \%$ des coûts bruts de formation. Ces dépenses doivent être relativisées car le coût brut s'amortit en moyenne largement - voire souvent intégralement - dans nombre d'entreprises, au fil des deux à trois années d'apprentissage, grâce à la contribution productive croissante de l'apprenti au cours de sa formation. Une étude sur les coûts et bénéfices de l'apprentissage menée régulièrement au BIBB estime à environ $12535 €$ par apprenti et par an le retour sur investissement, de sorte que les coûts nets s'élèvent à environ $5398 €$ par an et par apprenti (BIBB, 2015). Les coûts et produits de la formation varient en fonction des régions, de la taille de l'entreprise formatrice, du domaine de formation (commerce, artisanat, agriculture, professions libérales, service public), de la profession concernée, de l'utilisation d'atelier de formation et de la durée de formation (Jansen et al., 2015 $5^{5}$ ). La rémunération des apprentis varie entre $375 €$ en première année et $1300 €$ par mois en troisième année de formation en Allemagne, selon la branche, l'âge, le niveau de formation ou encore les conventions collectives spécifiques du secteur ${ }^{6}$. La rémunération moyenne s'élève en 2014 à $795 €(B I B B, 2015)$.

\footnotetext{
4. Par exemple, des jeunes dans des régions ayant des infrastructures insuffisantes, des jeunes ayant un besoin de support spécifique.

5. Étude réalisée sur les coûts de la formation duale et les pratiques de recrutement par le BIBB pour l'année 2012/2013 auprès de 3032 entreprises formatrices et 913 entreprises ne participant pas au système de formation duale.

6. Datenbank Ausbildungsvergütungen (base de données sur la rémunération des apprentis) :

https://www.bibb.de/de/12209.php
} 


\section{LA DÉFINITION DES BESOINS ET CONTENUS DES RÈGLEMENTS EN FORMATION DUALE}

Le développement ou l'actualisation des référentiels est une bonne illustration du rôle et de l'implication du monde professionnel dans le système allemand de formation professionnelle duale. Il s'agit d'un travail conjoint entre des spécialistes du BIBB, des experts représentant les partenaires sociaux et des représentants des Länder. Ce processus est administré par le BIBB. 155 métiers préparés par l'apprentissage ont vu leurs référentiels modifiés entre 2005 et 2015, parmi lesquels 24 nouvelles certifications ont vu le jour. En 2014, neuf référentiels ont été actualisés. Le référentiel ou règlement de formation (Ausbildungsordnung) fixe la dénomination de la profession, la durée de la formation, les savoir-faire, connaissances et capacités professionnels qui feront au minimum l'objet de la formation professionnelle (profil de la profession reconnue officiellement), les instructions concernant les programmes et les horaires requis pour inculquer les savoir-faire, connaissances et capacités professionnels (plan cadre de formation), les attentes pour satisfaire aux examens (Loi fédérale, article 5). Ces référentiels régissent ainsi les modalités concrètes de la formation professionnelle duale dans les entreprises et les écoles professionnelles. Ils sont à la base de l'éventail d'offres, du suivi et du soutien à la formation duale dans toute l'Allemagne.

Dans la plupart des cas, les employeurs, les associations patronales ou les syndicats identifient un besoin de création ou de modernisation d'un référentiel. Cela peut correspondre à l'émergence de nouveaux secteurs d'activité dans les entreprises ou à l'apparition de nouvelles technologies, par exemple en matière d'informatique ou de gestion de l'environnement. Ce besoin est généralement relayé auprès du ministère fédéral compétent. Ce dernier délivre un ordre de mission au BIBB. Le BIBB met en place un groupe d'experts composés de spécialistes du métier concerné, de représentants des entreprises (nommés par les chambres compétentes) et de représentants des syndicats. Ce groupe travaille pendant environ un an à l'actualisation du référentiel, et dix-huit moislen moyenne pour la création d'un référentiel. En respect du principe de subsidiarité en matière de formation professionnelle et d'éducation, deux sousgroupes sont établis pour travailler l'un avec le BIBB sur les aspects relevant de la formation en milieu de travail et l'autre sous la direction des acteurs représentant les Länder. Suite à cette phase de développement, un projet de règlement est établi qui, de façon complémentaire aux travaux des deux sous-groupes, s'attache à régler l'articulation des phases et contenus d'apprentissage entre les deux futurs lieux de formation (milieu de travail et école professionnelle). Cette ébauche complète du référentiel est soumise au comité principal du BIBB (Hauptausschuss) pour formuler une recommandation auprès du gouvernement fédéral. Le règlement de formation est ensuite publié au Journal officiel et la formation peut être offerte dès l'année scolaire suivante, au $1^{\text {er }}$ août (BIBB, 2014b). 


\section{LE MONDE PROFESSIONNEL COMME LIEU DE FORMATION}

Comme mentionné ci-dessus, la formation professionnelle duale s'effectue pour environ $70 \%$ de sa durée en milieu professionnel. Les chambres consulaires et les organisations patronales soutiennent l'effort des entreprises dans cette mission. Sa mise en place obéit à certaines normes prescrites par la loi sur la formation professionnelle. Ainsi, l'entreprise doit couvrir et être capable de former à toutes les tâches professionnelles fixées dans le règlement de formation ; elle doit également posséder l'équipement technique et un environnement favorable ; ce dernier concerne entre autres la gestion du personnel (sanitaire, vestiaire, etc.) et la mise à disposition du personnel qui sera en charge de l'accompagnement et de la formation de l'apprenti. L'entreprise est ainsi soumise à une accréditation comme entreprise formatrice réalisée par la chambre consulaire compétente. Si une entreprise n'est pas en mesure d'assurer la formation pour des différentes raisons telles que le manque d'équipements techniques ou technologiques ou que son processus de production ne permet pas la transmission de certains savoir-faire ou connaissances, l'entreprise peut faire appel à des programmes de formation qui se déroulent en dehors de l'entreprise, en centre inter-entreprises ou au sein de réseaux d'entreprises. Cette extension est soumise à un accord préalable de la chambre consulaire compétente.

La loi fédérale demande aux entreprises de sélectionner au sein de leur personnel des collaborateurs qui seront en charge de la formation professionnelle. En 2013, 85548 personnes ont passé l'examen d'accréditation pour être formateurs en entreprise dans les secteurs de l'industrie, du commerce, de l'artisanat, de l'agriculture et de la fonction publique (BIBB, 2015). Ces examens portent sur les aptitudes personnelles, pédagogiques et professionnelles du personnel en charge de la formation. Aucune personne ayant enfreint la loi ou n'étant pas autorisée à employer des enfants et des jeunes n'est autorisée à former en entreprise. Les coûts de cette formation varient en fonction des prestataires et peuvent ou non être pris en charge par l'entreprise. À ces coûts s'ajoutent pour les entreprises le temps de travail du formateur et du personnel mis à disposition pour la formation de l'apprenti. Dans les entreprises, l'équipe formatrice comprend souvent des formateurs accrédités en application de la législation mais également des salariés expérimentés exerçant cette activité de formation et d'accompagnement à titre accessoire.

\section{INSERTION SUR LE MARCHÉ DU TRAVAIL}

L'insertion des jeunes diplômés immédiatement après une formation duale en Allemagne n'est pas facile à décrire d'un point de vue statistique. Les chiffres disponibles ne couvrent qu'en partie la réalité. Nous avons à notre 
disposition le taux d'embauche par des entreprises et le taux de chômage après la formation initiale.

Pour l'année 2012, le taux d'embauche suite à une formation initiale duale s'élève à $66 \%$ en moyenne en Allemagne (BIBB, 2014a). Ce taux atteint $50 \%$ pour les entreprises formatrices de moins de dix salariés et plus de $75 \%$ pour les entreprises formatrices de plus de cinquante salariés. Beaucoup de grandes entreprises forment au-delà de leur besoin interne en main d'œuvre nouvelle. De façon générale, les entreprises considèrent la formation professionnelle comme un pré-recrutement ou, comme mentionné ci-dessus, comme un investissement d'avenir. Deux apprentis sur trois trouvent ainsi un emploi dans l'entreprise qui les a formés. En 2015, selon une étude du BIBB sur les pratiques de recrutement dans les domaines de la maintenance de véhicule et des soins infirmiers au cours des cinq dernières années, environ $55 \%$ des entreprises du secteur de la maintenance automobile indiquent qu'au moins $30 \%$ de leurs nouveaux ouvriers qualifiés sont des débutants ayant achevé une formation professionnelle duale (mécatronicien automobile). Seules $18 \%$ des entreprises n'ont pas recruté de débutants. Dans le domaine des soins infirmiers, environ $30 \%$ des entreprises n'ont pas recruté de débutants tandis que $26 \%$ des entreprises ont recruté au moins $50 \%$ de débutants parmi leurs nouveaux salariés (au niveau des ouvriers qualifiés) (Grollmann et al., 2016).

Le taux de chômage après une formation initiale était de $31 \%$ en moyenne en 2013 (BIBB, 2015). On observe des différences entre les régions, avec un taux plus élevé pour la partie orientale $(41 \%)$ que pour la partie occidentale du territoire. Ce taux porte sur les inscriptions au registre du chômage juste après la finalisation de la formation professionnelle. Nombre de jeunes s'inscrivent au chômage comme phase de transition avant de commencer des études supérieures, une formation continue (pour obtenir un diplôme de Meister) ou lorsqu'ils disposent d'une promesse d'embauche non encore réalisée en fin de formation. Ces statistiques nationales donnent une indication à un moment précis des parcours individuels professionnels. Les chiffres de l'OCDE indiquent que sur le long terme, $85 \%$ des personnes ayant entre 25 et 34 ans et un diplôme de formation professionnelle duale et $79 \%$ des personnes ayant entre 25 et 64 ans sont actives en Allemagne. La proportion des personnes ayant entre 15 et 29 ans sans emploi, éducation ou formation s'élève à $10 \%$, un chiffre inférieur à la moyenne des pays membres de l'OCDE (15\%) (OCDE, 2014).

Ces statistiques montrent dans leur ensemble que l'insertion sur le marché du travail pour les diplômés d'une formation duale fonctionne bien. Lorsque les jeunes ne sont pas employés par l'entreprise qui les a formés, la capacité d'absorption du marché du travail est une donne importante. 
$41 \%$ des entreprises estiment que le fait de soutenir la tradition de formation duale est un facteur important de leur motivation et $63 \%$ qu'il s'agit d'une tâche de responsabilité sociétale (Jansen et al., 2015). Par tradition, fondée à la fois sur l'expérience historique et sur la loi, les organisations du monde professionnel sont reconnues comme l'opérateur principal de la formation professionnelle initiale. C'est aux entreprises que revient l'initiative de recruter les apprentis, de s'engager en formation et de mettre en œuvre les moyens nécessaires pour la mener à bien. Leur engagement est largement motivé par leur besoin en main d'œuvre qualifiée qu'elles recrutent d'autant plus qu'elles connaissent bien les apprentis au bout de trois ans de formation. $83 \%$ des entreprises interrogées déclarent qu'elles forment ainsi leurs propres ouvriers et employés qualifiés qui resteront sur le long terme dans l'entreprise (Jansen et al., 2015). Les organisations syndicales sont également largement impliquées dans la définition et la mise en ouvre de la formation professionnelle duale. L'engagement des entreprises dans le système de formation professionnelle duale reste néanmoins un défi permanent, de leur nombre dépend largement le succès de la formation professionnelle. Le succès du système dual en Allemagne est également lié à la structuration du marché du travail et au fait que l'accès à certaines professions n'est ouvert qu'aux personnes titulaires d'un diplôme de formation professionnelle duale.

\section{BIBLIOGRAPHIE}

BIBB (2014a) : Datenreport zum Berufsbildungsbericht. Informationen und Analysen zur Entwicklung der beruflichen Bildung, BIBB Datenreport, Bonn: Bundesinstitut für Berufsbildung.

BIBB (ed.) (2014b) : Training regulations and how they come about, Schriftenreihe des Bundesinstituts für Berufsbildung, $6^{\mathrm{e}}$ édition révisée, Bonn: BIBB.

BIBB (2015) : Datenreport zum Berufsbildungsbericht. Informationen und Analysen zur Entwicklung der beruflichen Bildung, BIBB Datenreport, Bonn: Bundesinstitut für Berufsbildung.

GOUVERNEMENT FÉDÉRAL (2005) : Loi sur l'enseignement professionnel, Bonn.

GROLLMANN P. et al.(2016) : Muster betrieblicher Rekrutierungs- und Einarbeitungsprozesse in ausgewählten Ländern Europas, Abschlussbericht zu Forschungsprojekt 1.5.304 (JFP 2011), Laufzeit I/12-II/15, Bonn: Bundesinstitut für Berufsbildung.

JANSEN A. et al. (2015) : Ausbildung in Deutschland weiterhin investitionsorientiert - Ergebnisse der BIBB-Kosten-Nutzen-Erhebung 2012/13, BIBB-Report, vol. 1/2015, Bonn: Bundesinstitut für Berufsbildung.

LASSERRE R. (2014) : La formation professionnelle en Allemagne. Dynamiques socioéconomiques et capacités d'adaptation d'un système, Note du Cerfa, $\mathrm{n}^{\circ}$ 112, mai 2014.

MARTINOT B. (2015) : L'apprentissage, un vaccin contre le chômage des jeunes Plan d'action pour la France tiré de la réussite allemande. Institut Montaigne / METI, Paris.

OCDE (2014) : Education at a glance 2014: country note Germany, Paris : Éditions OCDE. STATISTISCHES BUNDESAMT (2015): Bildung und Kultur - Berufliche Schulen, Fachserie 11, reihe 2, Wiesbaden: Statistisches Bundesamt. 